\title{
Cylindrical Magnetorheological Fluid Variable Transmission Controlled by Shape-Memory Alloy
}

\author{
Song Chen, Kailin Jian, and Xianghe Peng \\ College of Resources and Environmental, Chongqing University, Chongqing 400044, China \\ Correspondence should be addressed to Song Chen, songchen1133@163.com
}

Received 15 June 2012; Accepted 2 September 2012

Academic Editor: Yan Yang

Copyright () 2012 Song Chen et al. This is an open access article distributed under the Creative Commons Attribution License, which permits unrestricted use, distribution, and reproduction in any medium, provided the original work is properly cited.

Centrifugal fan is an important component of a ventilation system in a nuclear power plant. In this paper, we proposed a magnetorheological speed-adjustment system controlled by shape-memory alloy for centrifugal fan. A theoretical analysis of the effect of the applied magnetic field on the viscoplastic flow between two cylinders in the speed-adjustment system is presented. The expressions for the velocity in viscoplastic flow and the torque transmitted by MR fluids are derived. A sliding mode SMA switch is proposed to modify the magnetic field acting on working gap under thermal effect. The results indicate that with the increases of applied magnetic field, the torque transmitted by MR fluid goes up rapidly.

\section{Introduction}

Centrifugal fans are widely used for transporting air in a ventilation system in a nuclear power plant. For different operation conditions of ventilating system, the rotational velocity of centrifugal fan needs to be adjusted to improve the operating efficiency of a ventilating system. In this paper, we propose a speed-adjustment system based on magnetorheological (MR) fluid and shape-memory alloy (SMA) for centrifugal fan.

MR fluids are the suspension of micron-sized, magnetizable particles in a carrier fluid. When exposed to a magnetic field, the rheological characteristics of MR fluids reversibly and instantaneously change from a free-flowing liquid to a semisolid with controllable yield strength. Altering the strength of the applied magnetic field precisely and proportionally controls the consistency or yield strength of the fluids $[1,2]$. Based on the mechanical characteristics, the fluids can be used in the controllable, energy-dissipating applications such as dampers $[3,4]$, valves $[5,6]$, and variable transmission devices [7-9].

An MR variable transmission device transmits torque by the shear stress of the MR fluids from the driving shaft to driven shell. The MR variable transmission device has the property that its transmitting torque changes quickly in response to an external magnetic field. A literature review indicates that the variable transmission devices that use MR fluids can be classified as having either disc type or cylindrical type [10]. In the disc type, the MR fluid within the activation region between two parallel circular plates is a circular plate. In the cylindrical type, the activated MR fluid between two concentric cylinders is an annular cylinder.

In order to rationally design the MR variable transmission devices, a designer should analyze the torque transmission ability. In this paper, Bingham model is used to describe the constitutive characteristics of MR fluids subject to an applied magnetic field. The operational model of the cylindrical type variable transmission device is established to derive the formula for the torque transmitted by MR fluids.

\section{Properties of MRF and SMA}

MR fluids can change their rheological characteristics by applying magnetic field. In the absence of an applied magnetic field, MR fluids exhibit a Newtonian-like behavior. However, upon application of a magnetic flied, the behavior of the controllable fluid is often represented as a Bingham fluid having a variable yield strength. In this model, the flow is governed by Bingham's equation [11]

$$
\begin{gathered}
\tau=\tau_{y}(H)+\eta \dot{\gamma} \quad \text { at } \tau \geq \tau_{y}(H), \\
\dot{\gamma}=0 \quad \text { at } \tau \leq \tau_{y}(H),
\end{gathered}
$$


where $\tau$ is the shear stress of fluid, $\tau_{y}(H)$ is the yield stress in response to an applied magnetic field $H, \eta$ is the viscosity of MR fluid with no applied magnetic field, and $\dot{\gamma}$ is the shear rate. Equations (1a) and (1b) indicate that for the shear stress exceeds the yield stress an MR material flows as a Newtonian fluid with a viscosity [12]. Otherwise it remains viscoelastic property. The yield stress $\tau_{y}(H)$ has a relationship with $H$ approximately as [13]

$$
\tau_{y}(H)=K H^{\beta},
$$

where $K$ and $\beta(1<\beta<2)$ are constants that determined by experiment.

SMAs are widely used as actuator materials [14, 15]. The thermoelastic martensite transformation of SMA can be expressed with one-dimensional constitutive equation [16]

$$
\dot{\sigma}=E \dot{\varepsilon}+\theta \dot{T}+\Omega \dot{\zeta}
$$

where $\sigma$ and $\varepsilon$ are SMA's stress and strain, respectively, $T$ is the temperature, $\zeta$ is the martensite volume fraction $(0 \leq \zeta \leq$ $1), E$ is the elastic modulus, $\theta$ is the elasticity coefficient for the heat, and $\Omega$ is the coefficient of phase change.

\section{Operational Principle}

The schematic of the cylindrical-type MR variable transmission is shown as Figure 1. The MR fluid is filled with the working gap between two concentric cylinders in the variable transmission. The MR variable transmission device depends on the shear stress of the MR fluid to transmits torque. In the absence of an applied magnetic field, MR fluids flow freely and exhibit Newtonian-like fluid behavior. The torque transmitted by the viscous stress of the MR fluid in the variable transmission is much smaller so that the suspended particles of the MR fluid cannot restrict the relative motion between the driving-cylinder and the driven-cylinder of the variable transmission. The variable transmission is in the state of separation. However, in the course of operation, a magnetic flux path is formed when electric current is put through the coil assembly. As a result, the MR particles are gathered to form chainlike structures, in the direction of the magnetic flux path. These chainlike structures increase the shear stress of the MR fluid. With the increase of the applied magnetic field, the shear stress developed by the MR fluid goes up rapidly. When the shear stress is large enough, the torque transmitted by the shear stress can join the driving cylinder with the driven cylinder. The amount of torque can be adjusted continuously by changing the input current. The current in coil can be modulated by a sliding mode SMA switch which alters the sliding distance under thermal effect, shown in Figure 2. The resistance of the circuit can be altering by using the sliding mode SMA switch under different temperature. The SMA helical spring working against a conventional steel spring (referred here as the "biasing" spring). At low temperatures, the steel spring is able to completely deflect the SMA spring to its compressed length. When increasing the temperature of the SMA spring, it expands, compressing the steel spring and moving the push rod.

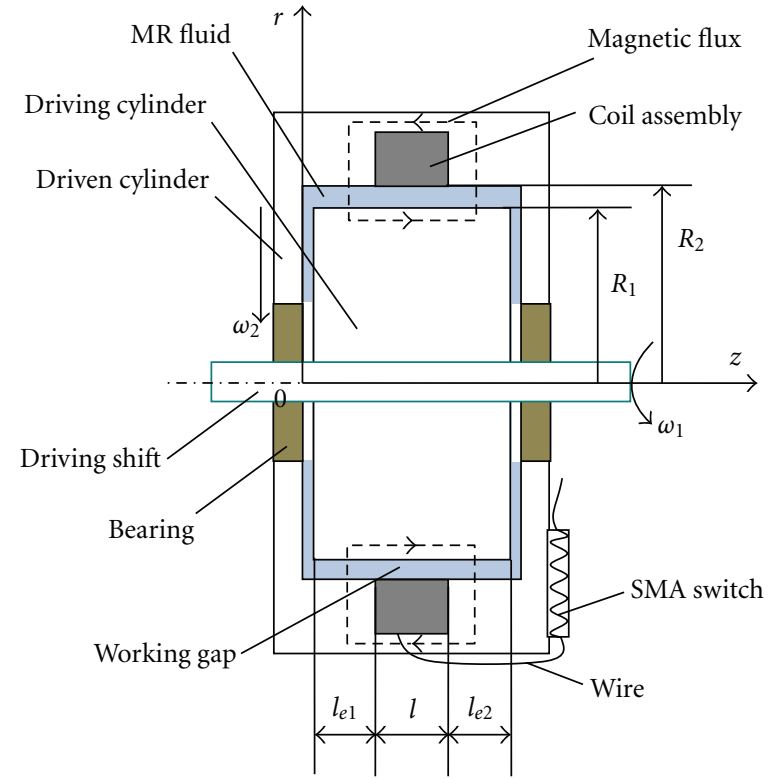

FIGURE 1: Schematic of a cylindrical MR variable transmission.

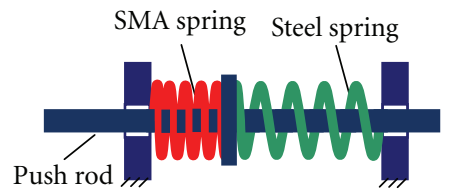

FIGURE 2: Operational principle of the SMA switch.

\section{Mathematic Model}

The output displacement of SMA switch can be expressed as follows [17]

$$
S(T)=\frac{\left(E(T)-E_{L}\right) \gamma_{L}}{\left(d / n \pi D^{2}\right) E(T)+\left(\left(F_{H}-F_{L}\right) / \Delta \delta\right)\left(E_{L} / F_{L}\right) \gamma_{L}},
$$

where $S(T)$ is the output displacement of SMA spring, $d$ represents the wire diameter, $D$ is the average diameter of the spring, $n$ is the number of turns in the spring, and $F_{L}, E_{L}$, and $\gamma_{L}$ are the axial load, shear modulus of SMA spring, and the strain at low temperature (in martensitic phase), respectively. $F_{H}$ is the axial load at high temperature, $\left(F_{H}-F_{L}\right) / \Delta \delta$ represents the spring constant of the conventional steel spring. $E(T)$ is the shear modulus of SMA at temperature $T$. In absence of stress, shear modulus of SMA can be expressed as (5) approximately:

$$
\begin{array}{r}
E(T)=E_{M}+\frac{E_{A}-E_{M}}{2}\left[1+\sin \phi\left(T-T_{m}\right)\right], \\
\text { when } M_{f} \leq T \leq A_{f},
\end{array}
$$

where $E_{M}$ and $E_{A}$ are the shear modulus of martensite and austenite, respectively, in the process of heating, $T_{m}=\left(A_{s}+\right.$ $\left.A_{f}\right) / 2, \phi=\pi /\left(A_{f}-A_{s}\right)$; in the process of cooling, $T_{m}=$ $\left(M_{s}+M_{f}\right) / 2, \phi=\pi /\left(M_{s}-M_{f}\right), M_{s}, M_{f}, A_{s}$, and $A_{f}$ are the 


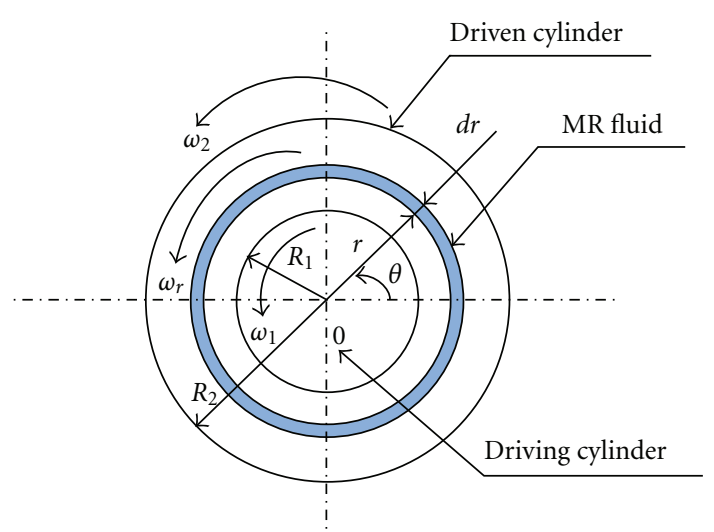

Figure 3: Circular flow of MR fluid between two cylinders.

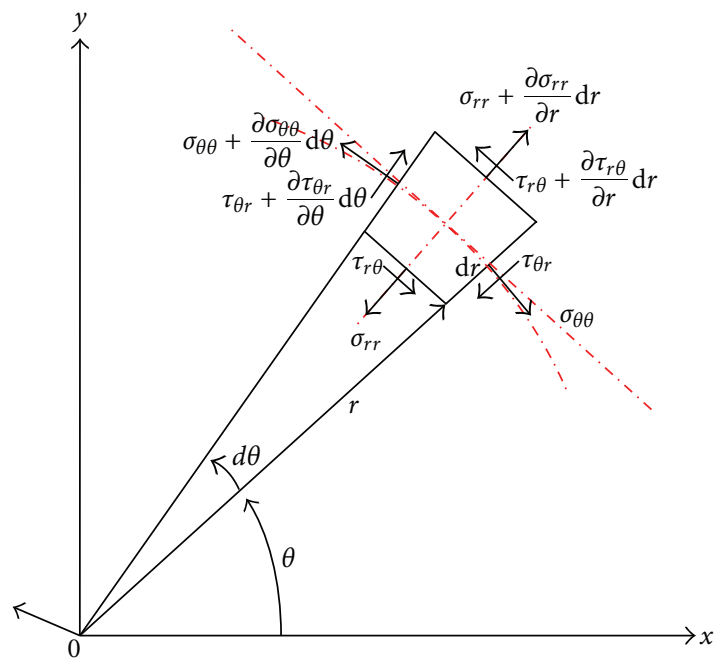

Figure 4: Stress analysis of differential element.

start and finish transformation temperatures of martensite and austenite, respectively.

The diagram of the operational mode of a cylindricaltype MR variable transmission is shown in Figure 3. In order to analyze the rheological behavior of MR fluids flow in the gap between two concentric cylinders in the cylindricaltype variable transmission, the following assumptions are given: the fluid is incompressible. There is no flow in radial direction and axial direction, but only tangential flow. The flow velocity of MR fluid is a function of radius. The pressure in the thickness direction of MR fluid is a constant. The strength of magnetic field in the working gap of the activation region is well distributed. In cylindrical coordinates $(r, \theta, z)$, the distribution of the flow velocity is

$$
v_{\theta}=r \omega(r), \quad v_{r}=0, \quad v_{z}=0,
$$

where $v_{\theta}, v_{r}$, and $v_{z}$ are the flow velocity of the fluid in the $\theta$-direction, $r$-direction, and $z$-direction, respectively. $\omega(r)$ is the rotation angular velocity of the fluid in the $\theta$-direction. The angular velocity $\omega(r)$ is the function of $r$-coordinate.

As shown in Figure 4, for the tangential shear flow of MR fluid between two cylinders, the force equilibrium equation in tangential can be derived from differential element of the fluid as follows:

$$
\begin{aligned}
& \left(\sigma_{\theta \theta}+\frac{\partial \sigma_{\theta \theta}}{\partial \theta} \mathrm{d} \theta\right) \mathrm{d} r d l \cos \frac{\mathrm{d} \theta}{2}-\sigma_{\theta \theta} \mathrm{d} r \mathrm{~d} l \cos \frac{\mathrm{d} \theta}{2} \\
& +\left(\tau_{\theta r}+\frac{\partial \tau_{\theta r}}{\partial \theta} \mathrm{d} \theta\right) \mathrm{d} r \mathrm{~d} l \sin \frac{\mathrm{d} \theta}{2}+\tau_{\theta r} \mathrm{~d} r \mathrm{~d} l \sin \frac{\mathrm{d} \theta}{2} \\
& +\left(\tau_{r \theta}+\frac{\partial \tau_{r \theta}}{\partial r} \mathrm{~d} r\right)(r+\mathrm{d} r) \mathrm{d} \theta \mathrm{d} l-\tau_{r} \theta \mathrm{d} \theta \mathrm{d} l=0
\end{aligned}
$$

where, $\sigma_{\theta \theta}$ is the compressive stress in the $\theta$-direction, $\tau_{\theta r}$ and $\tau_{r \theta}$ are the shear stress. According to the assumptions and ignore the parts of high-order, (7) can be simplified approximately as follows:

$$
\frac{\mathrm{d} \tau_{r \theta}}{\mathrm{d} r}+\frac{2 \tau_{r \theta}}{r}=0 .
$$

\section{Transmission Analysis}

Integrating the momentum equation (8), shear stress can be indicated as follows:

$$
\tau=\frac{c_{1}}{r^{2}}
$$

where $c_{1}$ is the integrating constant. The boundary conditions are

$$
\omega_{r}=\omega_{1} \quad \text { at } r=R_{1}, \quad \omega_{r}=\omega_{2} \quad \text { at } r=R_{2},
$$

where $R_{1}$ and $R_{2}$ are the radius of the inner and the outer cylinder, respectively. At two surfaces of $R_{1}$ and $R_{2}$, the shear stresses are, respectively:

$$
\tau_{1}=\frac{c_{1}}{R_{1}^{2}}, \quad \tau_{2}=\frac{c_{1}}{R_{2}^{2}} .
$$

The surface at $\tau_{y}(H)=c_{1} / R_{y}^{2}$, is defined as yield surface. $R_{y}$ is the radius of the cylindrical fluid at the yield surface. The field at $\tau>\tau_{y}(H)$ is yield, and the field at $\tau<\tau_{y}(H)$ is nonyield. At $\tau_{1}>\tau_{y}(H)>\tau_{2}$, the fluid of the field between $R_{1}$ and $R_{y}$ is viscous flow. Because the rotating speed decreases with the increases of the radius $r$, the shear rate $\dot{\gamma}$ in above equations ( $1 \mathrm{a}$ ) and ( $1 \mathrm{~b}$ ) can be calculated by the following:

$$
\dot{\gamma}=-r \frac{\mathrm{d} \omega_{r}}{\mathrm{~d} r},
$$

where $\mathrm{d} \omega_{r} / \mathrm{d} r$ is the rotating speed gradient in $r$-direction. Applying boundary conditions as $\omega_{r}=\omega_{1}$ at $r=R_{1}$, $d \omega_{r} / d r=0$ at $r=R_{y}$, the rotational speed can be obtained by (1a), (1b), (8), and (12) as follows:

$$
\begin{gathered}
\omega_{r}=\frac{R_{1}^{2} R_{y}^{2}}{R_{y}^{2}-R_{1}^{2}}\left[\left(\frac{R_{y}^{2}-r^{2}}{R_{y}^{2} r^{2}}\right)\left(\omega_{1}-\frac{\tau_{y}(H)}{\eta} \ln R_{1}\right)\right. \\
\left.+\left(\frac{r^{2}-R_{1}^{2}}{r^{2} R_{1}^{2}}\right)\left(\omega_{2}-\frac{\tau_{y}(H)}{\eta} \ln R_{y}\right)\right] \\
+\frac{\tau_{y}(H)}{\eta} \ln r .
\end{gathered}
$$


The fluid of the field between $R_{y}$ and $R_{2}$ is rotated by $\omega_{2}$ as a solid. The radius at the yield surface can be calculated by the following:

$$
R_{y}=\sqrt{\frac{T_{L}}{2 \pi \tau_{y}(H)}},
$$

where $T_{L}$ is the loading torque when MR fluid is nonyield, $2 \pi \tau_{y}(H) R_{1}^{2} \leq T_{L} \leq 2 \pi \tau_{y}(H) R_{2}^{2}$.

According to the above discussion, the flow has the following two particular conditions.

(1) At $\tau>\tau_{y}(H)$, the fluid within the gap between two cylinders is viscous flow. The rotational speed becomes

$$
\begin{aligned}
\omega_{r}=\frac{R_{1}^{2} R_{2}^{2}}{R_{2}^{2}-R_{1}^{2}} & {\left[\left(\frac{R_{2}^{2}-r^{2}}{R_{2}^{2} r^{2}}\right)\left(\omega_{1}-\frac{\tau_{y}(H)}{\eta} \ln R_{1}\right)\right.} \\
& \left.+\left(\frac{r^{2}-R_{1}^{2}}{r^{2} R_{1}^{2}}\right)\left(\omega_{2}-\frac{\tau_{y}(H)}{\eta} \ln R_{2}\right)\right] \\
& +\frac{\tau_{y}(H)}{\eta} \ln r .
\end{aligned}
$$

(2) At $\tau<\tau_{y}(H)$, the fluid within the gap between two cylinders is non-sipping. The rotational speed becomes

$$
\omega_{r}=\omega_{1}=\omega_{2} .
$$

The torque transmitted by the MR fluid can be calculated by the following:

$$
T=2 \pi r^{2} L \tau,
$$

where $T$ is the torque transmitted by the MR fluid, $L$ is the width of the working gap. In order to obtain the transmission torque, assuming that the MR fluid in working gap is yielded. Equations (1a), (1b), (12), (13), and (17) can be manipulated mathematically to yield:

$$
T=\frac{4 \pi L_{e} R_{1}^{2} R_{2}^{2} \ln \left(R_{2} / R_{1}\right)}{R_{2}^{2}-R_{1}^{2}} \tau_{y}(H)+\frac{4 \pi L R_{1}^{2} R_{2}^{2}\left(\omega_{1}-\omega_{2}\right)}{R_{2}^{2}-R_{1}^{2}} \eta,
$$

where $L_{e}$ is the effective width of the MR effect developed by the MR fluid.

\section{Computational Results and Discussions}

For this example, we using a typical MR fluid. Figure 5 shows the relation, obtained from the experiment, between the dynamic yield stress and the magnetic field strength for a typical MR fluid. From the figure we can find that the dynamic yield stress is proportional to the square of the magnetic field strength. MR fluid exhibits dynamic yield stresses of $0 \sim 48 \mathrm{kPa}$ for the applied magnetic field strength of $0 \sim 275 \mathrm{kAmp} / \mathrm{m}$. The ultimate strength of MR fluid is limited

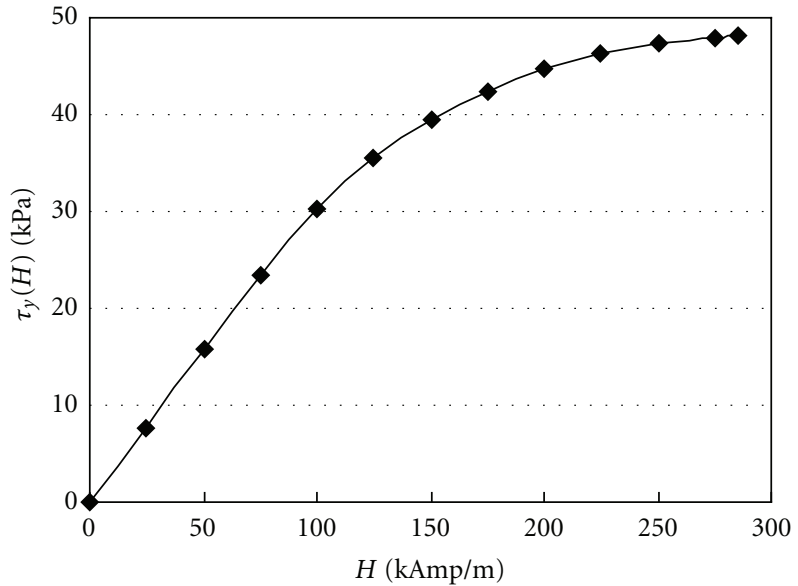

FIGURE 5: Yield stress versus magnetic field strength.

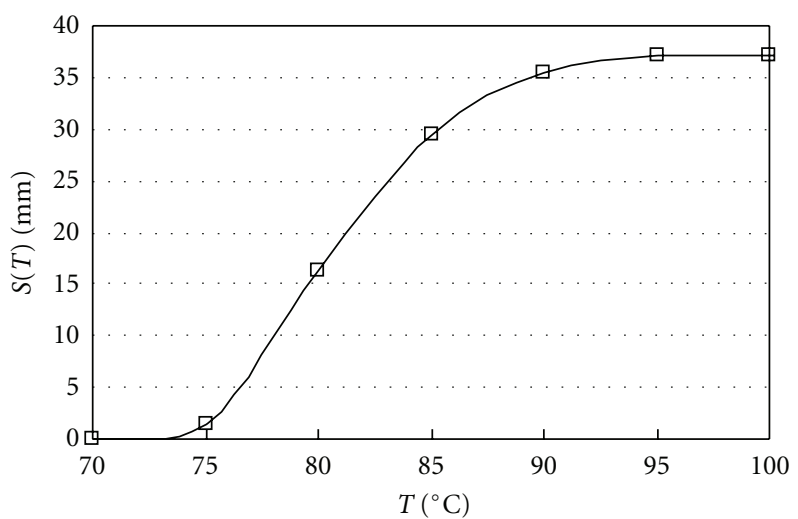

FIGURE 6: The output displacement versus temperature for SMA switch.

by magnetic saturation. The result shows that with the increase of the applied magnetic field strength, the dynamic yield stress goes up rapidly.

The effect of temperature in output displacement of SMA spring is shown in Figure 6. In this study, Ti-49.8 at.\% Ni SMA wire is used, its start and finish temperatures of the martensitic and austenitic phase transformation are $M_{s}=$ $78^{\circ} \mathrm{C}, M_{f}=50^{\circ} \mathrm{C}, A_{s}=74^{\circ} \mathrm{C}$ and $A_{f}=95^{\circ} \mathrm{C}$, respectively. The shear modulus of martensite and austenite are $G_{M}=$ $7.5 \mathrm{GPa}$ and $G_{A}=25 \mathrm{GPa}$, respectively. Assume that the low temperature shear strain is $\gamma_{L}=1.5 \%$, the wire diameter of SMA spring is $d=3 \mathrm{~mm}$, the average diameter of the spring is $D=13 \mathrm{~mm}$, the number of turns is $n=20$. As shown in Figure 5, the output displacement of SMA spring actuator increase with the increasing of temperature.

Figure 7 shows the relationship between the transmission torque with the gap size $h$ and inner radius $R_{1}$ for typical MR fluid in an applied magnetic field of $200 \mathrm{kAmp} / \mathrm{m}$. In this calculation, assume the following parameters are given: $\eta=0.092 \mathrm{~Pa} \cdot \mathrm{s}, L=100 \mathrm{~mm}, L_{e}=50 \mathrm{~mm}, \omega_{1}=200 \mathrm{rad} / \mathrm{s}$, $\omega_{2}=20 \mathrm{rad} / \mathrm{s}, h=R_{2}-R_{1}$. The results indicate that with the increase of the inner radius, the transmission torque developed by MR fluid goes up rapidly. 


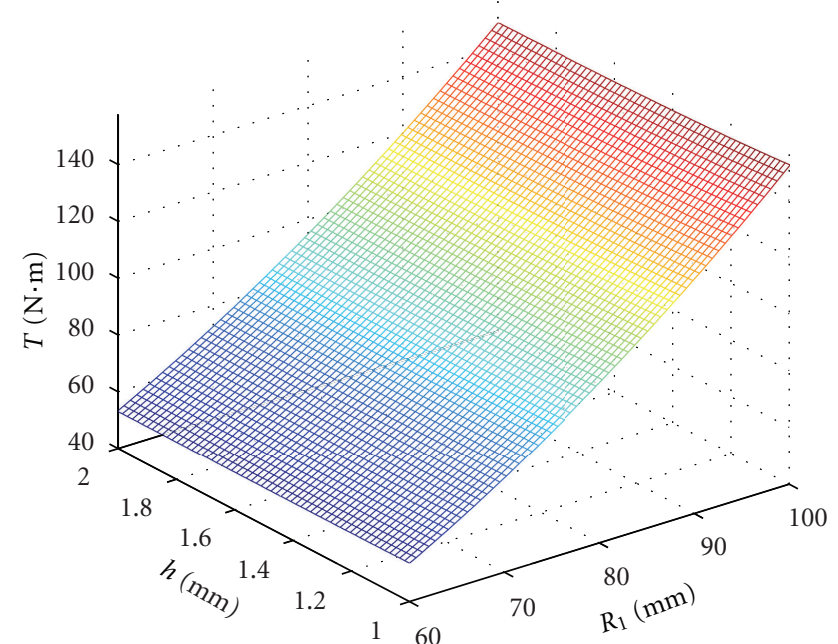

FIgURE 7: The transmission torque versus gap size $h$ and inner radius $R_{1}$.

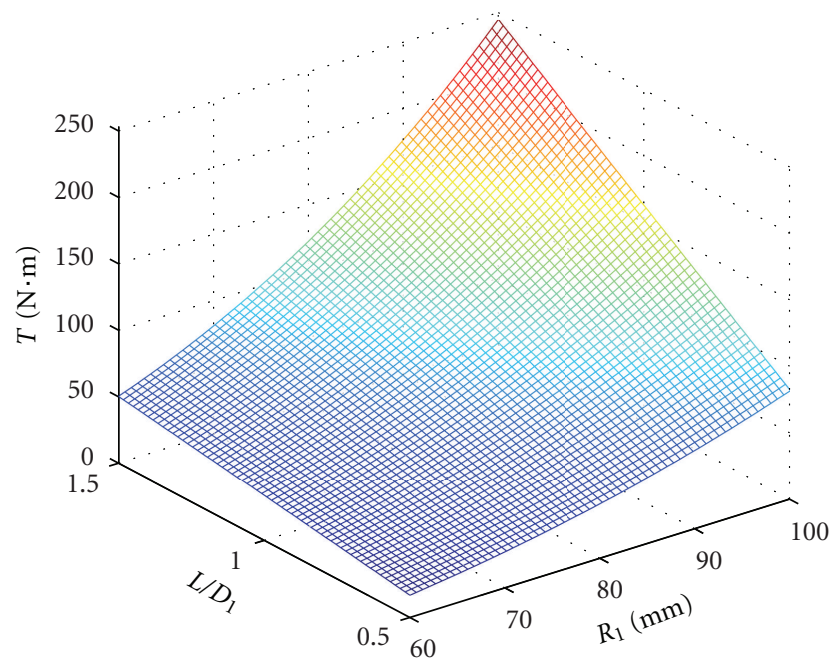

FIGURE 8: Torque versus breadth-diameter ratio $l$ and inner radius $R_{1}$.

Figure 8 shows the relationship between the transmission torque with the breadth-diameter ratio $l$ and inner radius $R_{1}$ for typical MR fluid in an applied magnetic field of $200 \mathrm{kAmp} / \mathrm{m}$. In this calculation, assume the following parameters are given: $\eta=0.092 \mathrm{~Pa} \cdot \mathrm{s}, \omega_{1}=200 \mathrm{rad} / \mathrm{s}, \omega_{2}=$ $20 \mathrm{rad} / \mathrm{s}, h=1 \mathrm{~mm}, l=L / D_{1}, D_{1}=2 R_{1}, L_{e}=(1 / 4) L$. The results indicate that with the increase of the breadthdiameter ratio, the transmission torque developed by MR fluid goes up rapidly.

Figure 9 shows the torque transmission ability at MR materials. The calculating parameters are: $\eta=0.092 \mathrm{~Pa} \cdot \mathrm{s}$, $R_{1}=90 \mathrm{~mm}, R_{2}=91 \mathrm{~mm}, L=100 \mathrm{~mm}, L_{e}=50 \mathrm{~mm}, q=$ $\omega_{1}-\omega_{2}$. In the absence of an applied magnetic field, the torque developed by the viscosity of MR fluids is $4.31 \mathrm{~N} \cdot \mathrm{m}$ when $q=200 \mathrm{rad} / \mathrm{s}$. When the magnetic field is applied, the torques are $46.25 \mathrm{~N} \cdot \mathrm{m}, 81.73 \mathrm{~N} \cdot \mathrm{m}$ and $106.95 \mathrm{~N} \cdot \mathrm{m}$ at the strength of magnetic field of $50 \mathrm{kAmp} / \mathrm{m}, 100 \mathrm{kAmp} / \mathrm{m}$ and

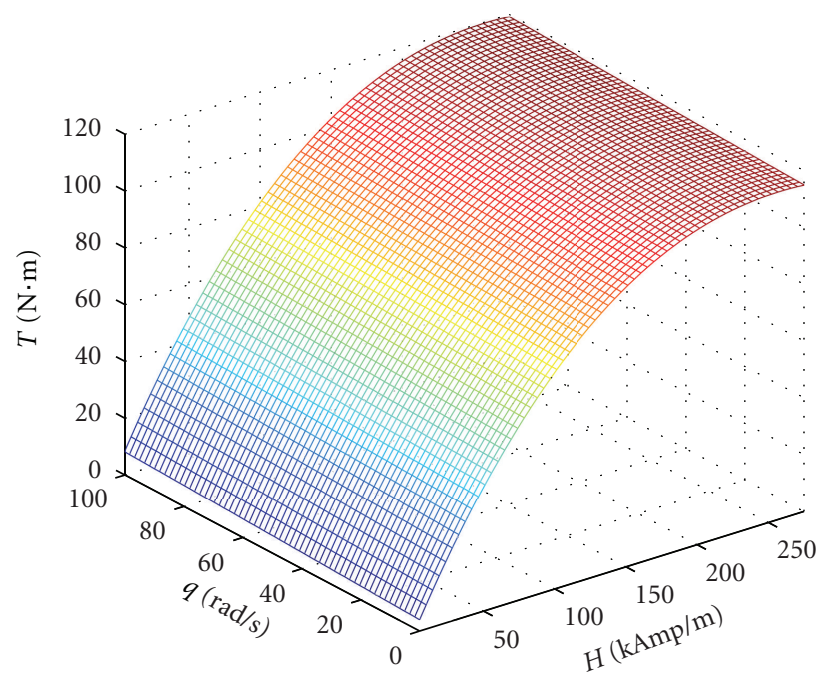

Figure 9: Torque versus magnetic field strength $H$ and rotative speed difference $q$.

$150 \mathrm{kAmp} / \mathrm{m}$, respectively, as the rotative speed difference $q=200 \mathrm{rad} / \mathrm{s}$. When MR fluid is saturated at the strength of magnetic field of $200 \mathrm{kAmp} / \mathrm{m}$, the torque is $117.37 \mathrm{~N} \cdot \mathrm{m}$. This torque is 27 times as much as the torque at zero magnetic field. The results indicate that with the increase of the applied magnetic field, the torque developed by MR clutch goes up rapidly.

\section{Conclusions}

An MR cylindrical-type variable transmission device depends on the shear stress of the MR fluid to transmits torque from the driving-shaft to driven-shell. The shear stress of MR fluid is increased with the increase of applied magnetic field strength. The applied magnetic field strength can be adjusted continuously by changing the output displacement of the sliding mode SMA switch under thermal effect. The transmission torque of MR fluid in the MR cylindrical-type variable transmission device is deeply influenced by magnetic field strength, breadth-diameter ratio $l$, inner radius $R_{1}$ and so on. After the structure is confirmed, The torque developed by MR fluid is increased rapidly with the increase of applied magnetic field strength.

\section{Acknowledgments}

Project 51175532 was supported by the National Natural Science Foundation of China and Key Project 2011BA4028 was supported by The Natural Science Foundation Project of CQ CSTC.

\section{References}

[1] W. Kordonski and S. Jacobs, "Model of magnetorheological finishing," Journal of Intelligent Material Systems and Structures, vol. 7, no. 2, pp. 131-137, 1996. 
[2] S. Jha and V. K. Jain, "Design and development of the magnetorheological abrasive flow finishing (MRAFF) process," International Journal of Machine Tools and Manufacture, vol. 44, no. 10, pp. 1019-1029, 2004.

[3] K. K. Ahn, D. Q. Truong, and M. A. Islam, "Modeling of a magneto-rheological (MR) fluid damper using a self tuning fuzzy mechanism," Journal of Mechanical Science and Technology, vol. 23, no. 5, pp. 1485-1499, 2009.

[4] X. Zhu, X. Jing, and L. Cheng, "Magnetorheological fluid dampers: a review on structure design and analysis," Journal of Intelligent Material Systems and Structures, vol. 23, no. 8, pp. 839-873, 2012.

[5] J. Huang, J. M. He, and J. Q. Zhang, "Viscoplastic flow of the MR fluid in a cylindrical valve," Key Engineering Materials, vol. 274-276, no. 1, pp. 969-974, 2004.

[6] A. M. Afonso, M. A. Alves, and F. T. Pinho, "Analytical solution of mixed electro-osmotic/pressure driven flows of viscoelastic fluids in microchannels," Journal of Non-Newtonian Fluid Mechanics, vol. 159, no. 1-3, pp. 50-63, 2009.

[7] J. Huang, L. Fu, and L. Zhong, "Analysis of a magnetorheological transmission for fan clutch," Advanced Materials Research, vol. 287-290, pp. 173-177, 2011.

[8] J. Z. Ma, G. C. Wang, and D. Zuo, "Geometric analysis in an MR fan clutch," Advanced Materials Research, vol. 239-242, pp. 1731-1734, 2011.

[9] E. S. Kim, J. W. Sohn, and S. B. Choi, "Temperature control using MR fan clutch for automotive application," Advanced Materials Research, vol. 79-82, pp. 79-82, 2009.

[10] J. Huang, J. Q. Zhang, Y. Yang, and Y. Q. Wei, "Analysis and design of a cylindrical magneto-rheological fluid brake," Journal of Materials Processing Technology, vol. 129, no. 1-3, pp. 559-562, 2002.

[11] J. D. Carlson and M. R. Jolly, "MR fluid, foam and elastomer devices," Mechatronics, vol. 10, no. 4, pp. 555-569, 2000.

[12] W. H. Li, H. Du, and N. Q. Guo, "Dynamic behavior of MR suspensions at moderate flux densities," Materials Science and Engineering A, vol. 371, no. 1-2, pp. 9-15, 2004.

[13] J. M. Ginder and L. C. Davis, "Shear stresses in magnetorheological fluids: role of magnetic saturation," Applied Physics Letters, vol. 65, no. 26, pp. 3410-3412, 1994.

[14] M. M. Barzegari, M. Dardel, A. Fathi, and M. Ghadimi, "Aeroelastic characteristics of cantilever wing with embedded shape memory alloys," Acta Astronautica, vol. 79, pp. 189-202, 2012.

[15] S. V. Raj, F. T. Calkins, I. Karaman, S. Miyazaki, R. D. Noebe, and R. Vaidyanathan, "Foreword physical and mechanical metallurgy of shape memory alloys for actuator applications," Metallurgical and Materials Transactions A, vol. 43, no. 8, article 2869, 2869.

[16] D. Z. Yang, Intelligent Materials and System, Tianjin University Press, Tianjin, China, 2000.

[17] J. Ma and P. Liu, "Design analysis in frictional fan clutch driven by SMA spring," Applied Mechanics and Materials, vol. 101102, pp. 7-10, 2012. 

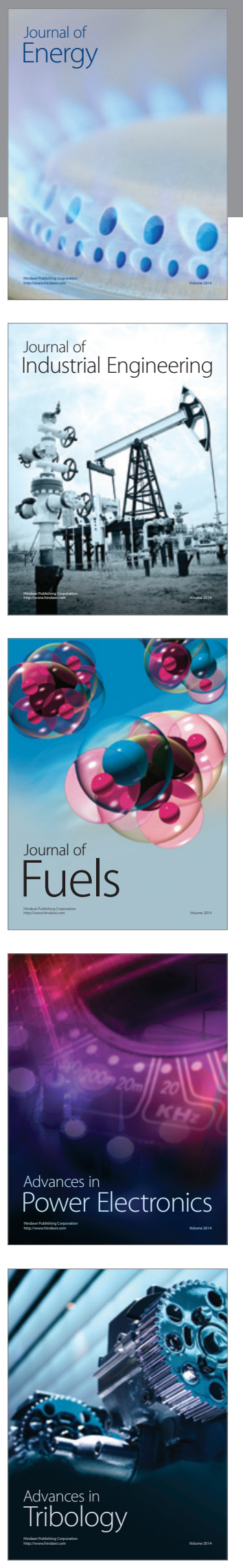
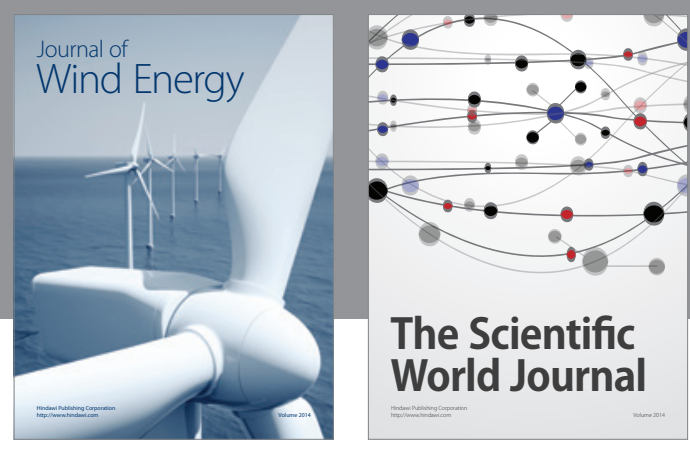

The Scientific World Journal

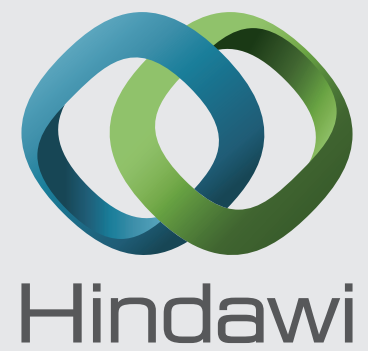

Submit your manuscripts at http://www.hindawi.com
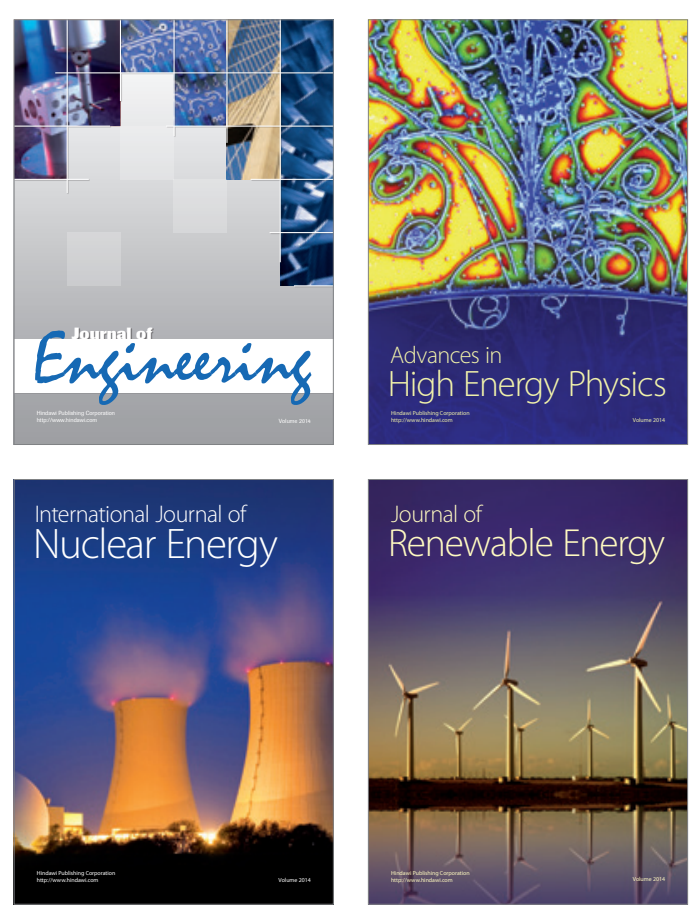

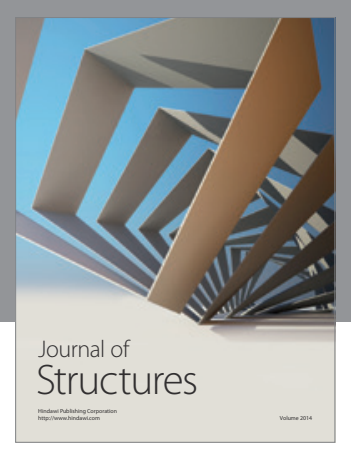

Rotating
Mechinery
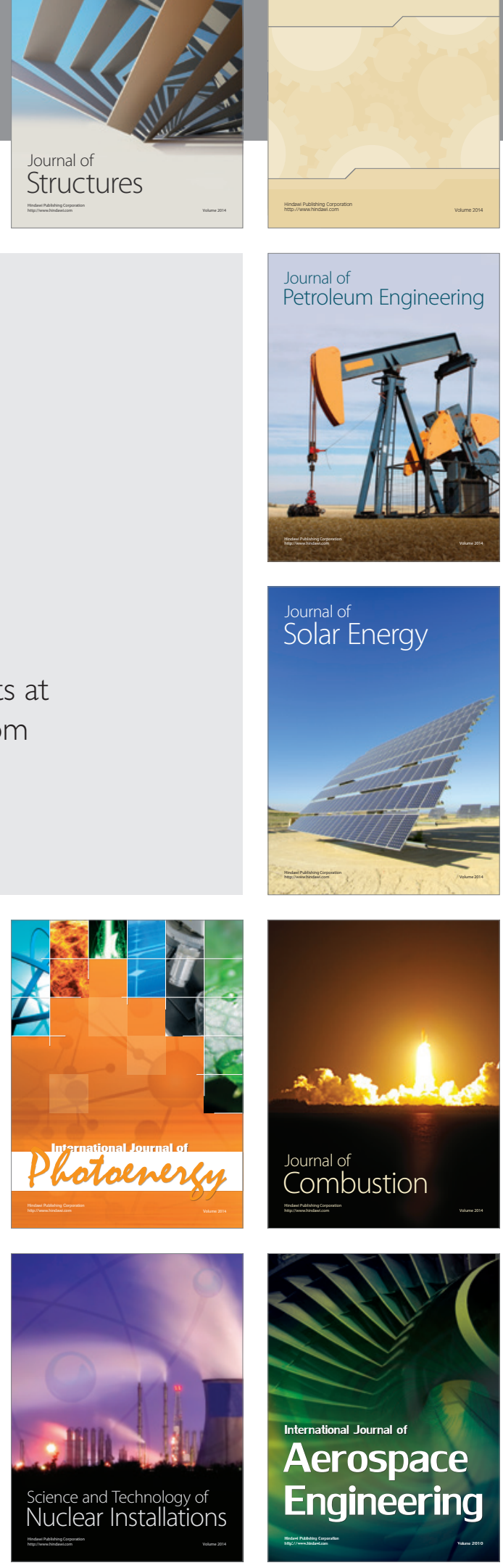